\title{
Impact of Electric Fields on Grain Boundary Atomic and Electronic Structures
}

Boyi Qu, Sean Russell and Klaus van Benthem

University of California, Davis, United States

Electric field assisted sintering, which includes Spark Plasma Sintering and Flash Sintering, has demonstrated the feasibility to accelerate densification of powder compacts, lower processing temperatures, and suppress or enhance grain growth [1]. A remaining fundamental question is how an applied electric field, in the absence of any appreciable current flow, affects grain boundary formation and the resulting interfacial microscopic degrees of freedom, i.e., local atomic interface structures.

For this study (100) twist grain boundaries of $\mathrm{SrTiO}_{3}$ were diffusion bonded at $1450^{\circ} \mathrm{C}$ either in the presence of absence of externally applied electrostatic fields. High-angle annular dark-field (HAADF) scanning electron microscopy (STEM) imaging was carried out in conjunction with energy-loss near-edge fine structure (ELNES) analysis to characterize the atomic and electronic grain boundary structures as a function of applied electric field. It was found that for nominal electrostatic field strength between $20 \mathrm{~V} / \mathrm{cm}$ and $170 \mathrm{~V} / \mathrm{cm} \mathrm{s}$ applied in the direction perpendicular to the grain boundary plane the interface expansion was only around $0.4 \mathrm{~nm}$, while a grain boundary width closer to $0.9-1.0 \mathrm{~nm}$ was detected after diffusion bonding in the absence of an applied electric field (see Figure 1). However, for field strength as high as $500 \mathrm{~V} / \mathrm{cm}$ and $1500 \mathrm{~V} / \mathrm{cm}$ applied parallel to the grain boundary plane changes in interface expansion between the negative and positive electrode side were observed. Initial ELNES results suggest a migration of oxygen vacancies within the applied electric field likely coupled with cation diffusion that may have caused unmixing and potential dissociation of $\mathrm{SrTiO} 3$ close to the negative electrode. Additional energydispersive X-ray spectroscopy and X-ray photoelectron spectroscopy experiments are underway to confirm this hypothesis.

The experimental results may suggest the feasibility to trigger grain boundary transitions with applied electrostatic fields during processing of insulating ceramics. 


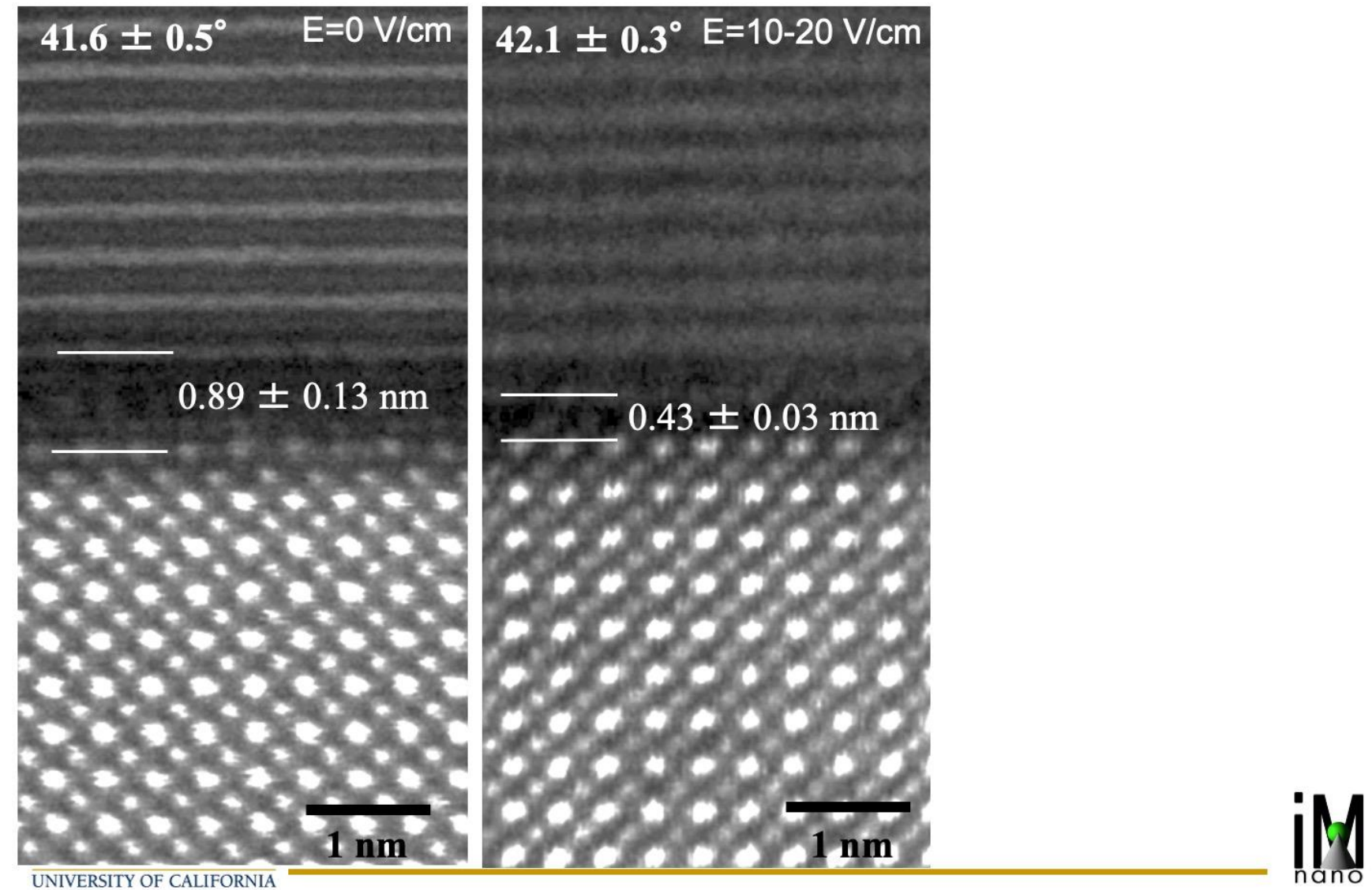

Figure 1. HAADF-STEM images of (100) twist grain boundaries in SrTiO3 diffusion bonded in the absence (left) and presence of an externally applied nominal electric field strength of 10-20 V/cm. Figure reproduced with permission from [2].

\section{References}

[1] W. Rheinheimer, M. Fülling, M.J. Hoffmann, Grain growth in weak electric fields in strontium titanate: Grain growth acceleration by defect redistribution, Journal of the European Ceramic Society36 (2016) 2773-2780.

[2] L.A. Hughes, M. Marple, K. van Benthem, Electrostatic fields control grain boundary structure in SrTiO3, Applied Physics Letters113 (2018) 041604.

[3] L.A. Hughes, $\underline{K}$. van Benthem, Effects of electrostatic field strength on grain boundary core structures in $\mathrm{SrTiO}_{3}$, Journal of American Ceramic Society 102 (2019) 4502-4510.

[4] This work was supported by the Army Research Office under award W911-NF-1610364 (program manager: Dr. Michael Bakas) and the National Science Foundation under award DMR-1836571. 